\title{
HULL SUBORDINATION AND EXTREMAL PROBLEMS FOR STARLIKE AND SPIRALLIKE MAPPINGS
}

BY

\author{
THOMAS H. MacGREGOR( ${ }^{1}$ )
}

ABSTRACT. Let $\mathcal{F}$ be a compact subset of the family $Q$ of functions analytic in $\Delta=\{z:|z|<1\}$, and let $\mathcal{Q}$ be a continuous linear operator of order zero on $Q$. We show that if the extreme points of the closed convex hull of $\mathcal{F}$ is the set $\left\{f_{0}(x z)\right\}(|x|=1)$, then $\mathfrak{L}(f)$ is hull subordinate to $\mathfrak{L}\left(f_{0}\right)$ in $\Delta$. This generalizes results of R. M. Robinson corresponding to families $\boldsymbol{F}$ of functions that are subordinate to $(1+z) /(1-z)$ or to $1 /(1-z)^{2}$. Families $\mathcal{f}$ to which this theorem applies are discussed and we identify each such operator $\mathcal{L}$ with a suitable sequence of complex numbers.

Suppose that $\Phi$ is a nonconstant entire function and that $0<\left|z_{0}\right|<1$. We show that the maximum of $\operatorname{Re}\left\{\Phi\left[\log \left(f\left(z_{0}\right) / z_{0}\right)\right]\right\}$ over the class of starlike functions of order $a$ is attained only by the functions $f(z)=z /(1-x z)^{2-2} a,|x|=1$. A similar result is obtained for spirallike mappings. Both results generalize a theorem of G. M. Golusin corresponding to the family of starlike mappings.

1. Introduction. In the first half of this paper we present a generalization of theorems proved by R. M. Robins on in [14] concerning the application of a linear operator to both sides of a subordination relation. We first need to introduce some notation and terminology.

Let $\mathbb{Q}$ denote the set of functions analytic in $\Delta=\{z:|z|<1\}$. Then $\mathscr{A}$ is a locally convex linear topological space with respect to the topology given by uniform convergence on compact subsets of $\Delta$. By a continuous linear operator we mean a mapping $\mathfrak{Q}$ of $\mathfrak{A}$ into $\mathfrak{Q}$ which is linear and continuous; briefly, $\mathfrak{Q}(a f+b g)=$ $a \mathfrak{L}(f)+b \mathscr{L}(g)$, and if $f_{n} \rightarrow f$ then $\mathscr{L}\left(f_{n}\right) \rightarrow \mathfrak{L}(f)$, each convergence being uniform on compact subsets of $\Delta$. An operator $\mathcal{L}$ is said to be of order zero if $\left.\{\varrho[f(w)]\}\right|_{w=x z}=\mathscr{Q}[f(x z)]$ for each $f$ in $\mathbb{Q}$ and for each complex number $x$ with Received by the editors February 8, 1973.

AMS (MOS) subject classifications (1970). Primary 30A32, 30A40, 30A42; Secondary $30 A 76$.

Key words and phrases. Subordination, hull subordination, continuous linear operator, functions with positive real part, Herglotz representation formula, extreme points, closed convex hull, univalent functions, starlike mapping, starlike functions of order $a$, spirallike functions, extremal problems, Krein-Milman theorem, probability measure, convex mapping, convex functions of order $a$, continuous linear functional.

(1) This research was partially supported by the National Science Foundation through grant GP-19709. 
$0<|x| \leq 1$. We say that $f$ is hull subordinate to $g$ in $\Delta$ if $f$ and $g$ belong to $\mathbb{A}$ and if $\{w: w=f(z),|z| \leq r\}$ is contained in the convex hull of $\{w: u=g(z),|z| \leq r\}$ for each $r(0<r<1)$. (For introductory facts about subordination we refer the reader to $[11$, pp. 226- ].)

One of the results proved by Robinson in [14] is the following theorem.

Theorem A. If $f(z)$ is subordinate to $g(z)=(1+z) /(1-z)$ in $\Delta$ and if $\mathfrak{L}$ is any continuous linear operator of order zero, then $\mathcal{L}(f)$ is bull subordinate to $\mathcal{L}(g)$ in $\Delta$.

The family of functions $f$ satisfying the hypothesis of Theorem $A$ is the same as the set $\mathcal{P}$ of functions in $\mathscr{Q}$ that satisfy $\operatorname{Re} f(z)>0$ and $f(0)=1$. Robinson obtained the conclusion of Theorem A more generally when $g$ is any conformal mapping of $\Delta$ onto a half-plane. He also proved such a theorem for functions that are subordinate to $g(z)=1 /(1-z)^{2}$ in $\Delta$.

Theorem 1 in this paper generalizes these results. Let $\mathcal{F} \mathcal{F}$ denote the closed convex hull of any subset $\mathcal{F}$ of $\mathfrak{Q}$, and let $\mathbb{E} \mathscr{F} \mathcal{F}$ denote the extreme points of $\mathcal{S C F}_{\text {(see }}$ 4, Chapter 5] for basic definitions and results). We show that if $\mathcal{F}$ is any compact subset of $\mathbb{Q}$ such that $\mathbb{E} \mathcal{F} \mathcal{F}=\left\{f: f(z)=f_{0}(x z),|x|=1\right\}$, then $\mathscr{Q}(f)$ is hull subordinate to $\mathfrak{L}\left(f_{0}\right)$ for each $f$ in $\mathfrak{F}$, where $\mathfrak{L}$ is any continuous linear operator of order zero. This generalizes Theorem A because the functions $f(z)=$ $(1+x z) /(1-x z),|x|=1$, are precisely the extreme points of $\mathcal{P}$. This is a wellknown consequence of the Herglotz representation formula for $\mathcal{P}$.

Our proof of Theorem 1 is quite easy, and it puts such facts in a simple general setting. This is particularly useful because the hypotheses hold for a number of interesting families of analytic functions, as has been verified quite recently. We indicate such families, most of which concern classes of univalent functions.

The diversity of applications of Theorem 1 is also due to the abundance of continuous linear operators of order zero. In [14] Robinson was particularly concerned with the operators given by

$$
z f^{\prime}(z), \quad[z f(z)]^{\prime}, \quad \int_{0}^{z} \frac{f(w)-f(0)}{w} d w, \quad \frac{1}{z} \int_{0}^{z} f(w) d w .
$$

Another interesting operator is the $n$th partial sum of the power series of a function in $\mathbb{Q}$. The scope of applications of Theorem 1 is further clarified by Theorem 2 , which identifies each continuous linear operator of order zero with a suitable sequence.

The second half of this paper concerns extremal problems for classes of univalent functions. Let $S$ denote the subset of $\mathfrak{Q}$ of functions univalent in $\Delta$ that satisfy $f(0)=0$ and $f^{\prime}(0)=1$. Also let $S t$ denote the subset of $S$ of starlike 
mappings-that is, $f(\Delta)$ is starlike with respect to $w=0$. We generalize the following theorem proved by G. M. Golusin in [5].

Theorem B.(2) Let $\Phi$ be any nonconstant entire function and suppose that $0<\left|z_{0}\right|<1$. Then, the maximum of either

$$
\operatorname{Re}\left\{\Phi\left[\log \frac{f\left(z_{0}\right)}{z_{0}}\right]\right\} \text { or }\left|\Phi\left[\log \frac{f\left(z_{0}\right)}{z_{0}}\right]\right|
$$

over the class $S_{t}$ is attained only for a function of the form $f(z)=z /(1-x z)^{2}$, $|x|=1$.

Golusin's proof of Theorem B depends on a variational method for St obtained by variations of a monotone function. In Theorem 3 we prove the analogous result for the class $S_{t}(\alpha)$ of starlike functions of order $\alpha$. This family was introduced by M. S. Robertson in [12] and we recall that $f \in S_{t}(\alpha)$ if $f \in \mathbb{Q}, f(0)=0, f^{\prime}(0)=1$ and $\operatorname{Re}\left\{z f^{\prime}(z) / f(z)\right\}>\alpha$ for $|z|<1$. We note that $a<1$, St $(0)=S_{t}$ and $S_{t}(\alpha) \subset S$ if $\alpha \geq 0$. Our argument used to prove Theorem 3 depends on several applications of the basic ideas of subordination and Theorem $A$.

The argument is equally adapted to prove a similar result for the class $S p(\alpha)$ of spirallike functions with parameter $\alpha$. This subclass of $\mathfrak{A}$ is defined by the conditions $f(0)=0, f^{\prime}(0)=1$ and $\operatorname{Re}\left\{e^{i a} z f^{\prime}(z) / f(z)\right\}>0$ for $|z|<1$. Here, $a$ is real and $|\alpha|<\pi / 2$. We note that $S_{p}(0)=S_{t}$ and $S_{p}(\alpha) \subset S$ as proved by L. Špaček in [15]. After writing this paper, we learned that the results about $S_{t}(\alpha)$ and $S_{p}(\alpha)$ (Theorems 3 and 4) were obtained earlier by B. Pinchuk, On starlike and convex functions of order $\alpha$, Duke Math. J. 35 (1968), 721-734. MR 37 \#6454; see Theorems 1 and 14 in the case $n=1$. Pinchuk's arguments use the variational methods of Golusin. The arguments we present are different and are quite simple and useful. We shall indicate other families for which such extremal problems can be resolved by arguments involving subordination. This approach also was used by M. S. Robertson in [13] with regard to the extremal problem of minimizing $\min |z|={ }_{r} \operatorname{Re}\{\Phi(q(z))\}$ where $q(z)=z^{-1} \int_{0}^{z} p(w) d w$ and $p$ varies in $\mathcal{P}$.

2. Hull subordination, extreme points, and continuous linear operators of order zero.

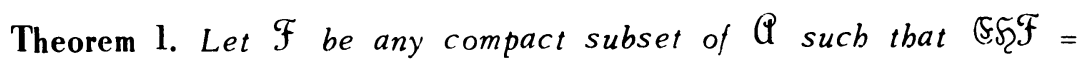
$\left\{f: f(z)=f_{0}(x z),|x|=1\right\}$. If $\mathcal{Q}$ is a continuous linear operator of order zero, then $\mathfrak{L}(f)$ is bull subordinate to $\mathfrak{L}\left(f_{0}\right)$ for each $f$ in $\mathfrak{F}$.

(2) This theorem was demonstrated by Golusin with an added restriction that was shown to be unnecessary by W. E. Kirwan in [10]. 
Proof. (We note that since $\mathcal{F}$ is compact, $\mathbb{E}[\mathcal{F} \mathcal{F} \subset \mathcal{F}[4$, p. 440] and thus our hypothesis implies that each function $f_{0}(x z),|x|=1$, belongs to $\mathcal{F}$.) We shall prove the assertion more generally for functions belonging to $\mathcal{F C F}_{\mathcal{F}}$. Because $\mathcal{F}$ is compact, the Kre ̌n-Milman theorem [4, p. 440] and our hypothesis on $(5 \mathcal{F F}$ imply that $\mathcal{F} \mathcal{F}$ is the same as the set of functions

$$
f(z)=\int_{X} f_{0}(x z) d \mu(x)
$$

where $\mu$ varies over the probability measures on $X=\{x:|x|=1\}$. If $\mathcal{L}$ is a continuous linear operator then equation (2) implies that

$$
\mathcal{L}[f(z)]=\int_{X} \mathcal{L}\left[f_{0}(x z)\right] d \mu(x) .
$$

To prove this, note that the integral in (2) may be uniformly approximated in $|z| \leq r(0<r<1)$ by a finite sum obtained by approximating $\mu$ by a convex combination of point masses. We next use the linearity of $\mathscr{Q}$ and finally the continuity of $\mathcal{L}$.

Because $\mathcal{L}$ is of order zero, equation (3) may be expressed as

$$
\mathcal{L}[f(z)]=\left.\int_{X}\left\{\mathscr{L}\left[f_{0}(w)\right]\right\}\right|_{w=x z} d \mu(x) .
$$

Equation (4) shows that the number $\mathfrak{\complement}[f(z)]$ is an average with total weight one of the values of $\mathscr{L}\left[f_{0}(w)\right]$ for $|w|=|z|$. Thus, $\mathfrak{L}[f(z)]$ is contained in the convex hull of the values of $\mathscr{L}\left[f_{0}(w)\right]$ for $|w| \leq|z|$, and so $\mathscr{L}(f)$ is hull subordinate to $\mathfrak{L}\left(f_{0}\right)$ for each $f$ in $\mathfrak{S} \mathcal{F}$.

Remarks and applications of Theorem 1. 1. When applied to 5ृF, Theorem 1 is sharp in the following sense. If $0<r<1$ and if $w_{1}$ is any point in the convex hull of the set $W=\left\{w: w=\mathfrak{L}\left[f_{0}(z)\right],|z| \leq r\right\}$ then there is a function $f$ in $\mathscr{S}_{\mathcal{F}}$ and a number $z_{1}$ so that $\mathscr{\varrho}\left[f\left(z_{1}\right)\right]=w_{1}$ and $\left|z_{1}\right| \leq r$. This is clear if $w_{1} \in W$, and

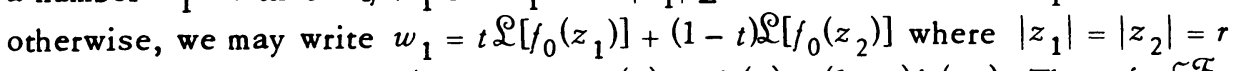
and $0<t<1$. Let $x=z_{2} / z_{1}$ and set $f(z)=t f_{0}(z)+(1-t) f_{0}(x z)$. Then $f \in \mathcal{S} \mathcal{F}$ and $\mathscr{L}\left[\left(z_{1}\right)\right]=t \mathfrak{L}\left[f_{0}\left(z_{1}\right)\right]+(1-t) \mathcal{L}\left[f_{0}\left(z_{2}\right)\right]=w_{1}$.

2. A result of D. Brannan, J. Clunie and W. Kirwan in [1] implies that if $\mathcal{F}$ is the set of functions in $\mathbb{Q}$ that are subordinate to $f_{0}(z)=[(1+c z) /(1-z)]^{p}$ in $\Delta(|c| \leq 1, p \geq 1)$, then $\mathbb{E} \mathcal{F} \mathcal{F}=\left\{f: f(z)=f_{0}(x z),|x|=1\right\}$. This generalizes the earlier work of L. Brickman, D. Wilken and this author in [2] corresponding to the cases $c=0$ and $p=1,2,3, \cdots$. It provides interesting families for which Theorem 1 is applicable. The case $c=1, p=1$ corresponds to Theorem $A$ and the case $c=0, p=2$ corresponds to the result of Robinson quoted earlier.

3. Let $K(\alpha)$ denote the class of convex functions of order $a$ introduced by M. S. Robertson in [12]. Recall that $f \in K(\alpha)$ if $f \in \mathbb{Q}, f(0)=0, f^{\prime}(0)=1$ and $\operatorname{Re}\left\{z f^{\prime \prime}(z) / f^{\prime}(z)+1\right\}>a(|z|<1)$. In [3] it was shown that 


$$
E_{5} S_{t}(\alpha)=\left\{f: f(z)=z /(1-x z)^{2-2 \alpha},|x|=1\right\}
$$

and that

$$
\text { EF } K(\alpha)=\left\{g: z g^{\prime}(z)=f(z), f \in \text { ES } S_{2} S_{t}(\alpha)\right\} \text {. }
$$

Theorem 1 is therefore applicable to the set $\{f(z) / z\}$ where $f \in S_{t}(\alpha)$ or where $f \in K(\alpha)$.

4. In [6] and [8] D. J. Hallenbeck obtained results which show that other families exhibit the situation described in 3. For example, if $C(\alpha)$ denotes the subset of $\mathbb{Q}$ such that $f(0)=0, f^{\prime}(0)=1$ and $\operatorname{Re} f^{\prime}(z)>a(|z|<1)$ then

$$
\mathfrak{E}[C(\alpha)]=\{f: f(z)=(2 \alpha-1) z+((2 \alpha-2) / x) \log (1-x z),|x|=1\} .
$$

Again, Theorem 1 is applicable to the class $\{f(z) / z\}$ where $f \in C(\alpha)$.

A generalization of this situation is the following fact: if $\mathcal{L}$ is any linear homeomorphism of $\mathfrak{F}$ onto $\mathcal{F}^{*}$ then $\left(\mathfrak{F} \mathcal{F}_{\mathcal{F}} *=\left\{f^{*}: f^{*}=\mathfrak{L}(f), f \in\left(\mathfrak{F} \mathcal{F}_{\mathcal{F}}\right\}\right.\right.$. Thus, if $\mathcal{F}$ is a family that satisfies Theorem 1 then so is $\mathcal{F} *$. If, for example, we begin with $\mathcal{P}$ and vary $\mathcal{L}$ we obtain a variety of families satisfying the theorem. The homeomorphism for the family $C(\alpha)$ is given by $\mathscr{\varrho}[f(z)]=z^{-1} \int_{0}^{z} f(w) d w$ and is applied to the family $a+(1-\alpha) \mathcal{P}$.

5. We are not interested in solving specific problems here but rather in indicating the range of applicability of Theorem 1 . Whenever its hypotheses hold, then the determination of the region of variability of $\left\{\mathscr{Q}\left[f\left(z_{0}\right)\right]\right\}$ with $f$ varying in $\mathfrak{5} \mathcal{F}$ is reduced to the specific problem of examining the set $\left\{\mathscr{Q}\left[f_{0}(z)\right]\right\}$ where $|z| \leq\left|z_{0}\right|$. Various extremal problems are hereby reduced to certain specific computations. A classical example of this is the fact that in order to prove that the partial sums of a function in $\mathcal{P}$ have a positive real part for $|z|<1 / 2$ one merely needs to show that $\operatorname{Re}\left\{1+2 z+2 z^{2}+\cdots+2 z^{n}\right\}>0$ for $|z|<1 / 2(n=1,2, \ldots)$. Some interesting problems were resolved in [14] by Robinson using Theorem $A$ and the analogous result with $g(z)=1 /(1-z)^{2}$. The problems which can be so solved are further indicated through our next theorem.

Theorem 2. Let $\&$ be a continuous linear operator of order zero. Then there is a sequence $\left\{b_{n}\right\}(n=0,1,2, \ldots)$ of complex numbers such that

$$
\varlimsup_{n \rightarrow \infty}\left|b_{n}\right|^{1 / n} \leq 1
$$

and if

$$
f(z)=\sum_{n=0}^{\infty} a_{n} z^{n} \quad(|z|<1)
$$


then

$$
\mathcal{Q}[f(z)]=\sum_{n=0}^{\infty} a_{n} b_{n} z^{n} .
$$

Conversely, if a sequence $\left\{b_{n}\right\}$ satisfies (5) then the operator $\mathcal{E}$ defined through

(6) and (7) defines a continuous linear operator of order zero.

Proof. Let $\mathcal{Q}$ be a continuous linear operator of order zero. Suppose that $f \in \mathbb{Q}$ and is given by (6). Then, the linearity and continuity of $\mathcal{Q}$ imply that

$$
\mathcal{L}[f(z)]=\sum_{n=0}^{\infty} a_{n} \varrho\left[z^{n}\right] \text {. }
$$

If we set $\mathscr{L}\left[z^{n}\right]=\sum_{k=0}^{\infty} a_{k, n} z^{k}$ and $\mathfrak{L}[f(z)]=\sum_{n=0}^{\infty}{ }^{c} z_{n}$, then (8) implies that

$$
c_{n}=\sum_{k=0}^{\infty} a_{k} \alpha_{n, k} \text {. }
$$

Also, if $\mathscr{L}[f(x z)]=\sum_{n=0}^{\infty} d_{n} z^{n}(|x| \leq 1)$, then $d_{n}=\sum_{k=0}^{\infty} a_{k} x^{k} \alpha_{n, k}$. Since $\mathcal{L}$ is of order zero and $\left.\{\mathfrak{Q}[f(w)]\}\right|_{w=x z}=\sum_{n=0}^{\infty} c_{n} x^{n} z^{n}$ we conclude that $d_{n}=c_{n} x^{n}$ $(n=0,1,2, \cdots,|x| \leq 1)$; that is,

$$
\sum_{k=0}^{\infty} a_{k} a_{n, k} x^{k}=\left\{\sum_{k=0}^{\infty} a_{k} a_{n, k}\right\} x^{n} .
$$

Since (10) holds for $|x| \leq 1$ the left-hand side must reduce to a monomial only involving a term of degree $n$. Therefore, $a_{k} a_{n, k}=0$ for $k \neq n$. This conclusion holds no matter what function $f$ in $\mathfrak{Q}$ we apply the argument to and so by first choosing such an $f$ with $a_{k} \neq 0$ for all $k$ (for example, $f(z)=1 /(1-z$ ) will do) we conclude that $a_{n, k}=0$ if $k \neq n$. If we set $b_{n}=a_{n, n}$ then $\mathscr{L}\left[z^{n}\right]=b_{n} z^{n}$ and so $\mathscr{L}[f(z)]=\sum_{n=0}^{\infty} a_{n} b_{n} z^{n}$. In order to prove that inequality (5) holds for this sequence $\left\{b_{n}\right\}$ we observe that since $f(z)=1 /(1-z)$ is in $\mathbb{A}$ then $\mathscr{Q}[f(z)]=$ $\sum_{n=0}^{\infty} b_{n} z^{n}$ must be analytic in $\Delta$. Thus, by Hadamard's formula for the radius of convergence of a power series, $R=1 / \overline{\lim }_{n \rightarrow \infty}\left|b_{n}\right|^{1 / n} \geq 1$, and so (5) holds.

Conversely, suppose that the sequence $\left\{b_{n}\right\}$ satisfies (5) and let $\mathcal{L}$ be defined by (6) and (7). If $f \in \mathbb{Q}$ then, again appealing to Hadamard's formula, the series in (7) also belongs to $\mathfrak{A}$ for

$$
\varlimsup_{n \rightarrow \infty}\left|a_{n} b_{n}\right|^{1 / n} \leq \varlimsup_{n \rightarrow \infty}\left|a_{n}\right|^{1 / n} \cdot \varlimsup_{n \rightarrow \infty}\left|b_{n}\right|^{1 / n} \leq 1 .
$$

It is easy to see that $\mathcal{Q}$ is the linear and of order zero. To show that $\mathcal{Q}$ is continuous, let the sequence $\left\{f_{k}\right\}$ belong to $\mathcal{Q}$ and converge to $f$ uniformly on com-

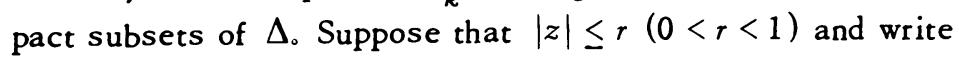




$$
f_{k}(z)=\sum_{n=0}^{\infty} a_{n, k} z^{n} \text { and } f(z)=\sum_{n=0}^{\infty} a_{n} z^{n}
$$

Then,

$$
\begin{aligned}
& \left|\mathscr{E}\left[f_{k}(z)\right]-\mathscr{L}[f(z)]\right|=\left|\sum_{n=0}^{\infty}\left(a_{n, k}-a_{n}\right) b_{n} z^{n}\right| \\
& \leq \sum_{n=0}^{N}\left|a_{n, k}-a_{n}\right|\left|b_{n}\right| r^{n}+\sum_{n=N+1}^{\infty}\left|a_{n, k} b_{n}\right| r^{n}+\sum_{n=N+1}^{\infty}\left|a_{n} b_{n}\right| r^{n} .
\end{aligned}
$$

The last two sums can be made small by choosing $N$ sufficiently large because $\mathscr{L}\left[f_{k}(z)\right]$ and $\mathscr{L}[f(z)]$ are analytic in $\Delta$. With $N$ so chosen then the first sum can be made small for sufficiently large $k$, for there are only finitely many terms and $a_{n, k} \rightarrow a_{n}$ as $k \rightarrow \infty$ since $f_{k} \rightarrow f$ uniformly in $|z| \leq r$.

Remark. As an illustration of The orem 2 we note that the operators defined by equation (1) are described by the respective sequences: $\{0,1,2,3, \ldots\}$, $\{1,2,3, \ldots\},\{0,1,1 / 2,1 / 3, \cdots\}$, and $\{1,1 / 2,1 / 3, \cdots\}$. The operator defined by the $n$th partial sum of a power series is given by the sequence that has 1 for its first $n+1$ terms and 0 for the remaining terms.

3. Extremal problems for starlike and spirallike mappings. In this section we obtain two generalizations of Theorem B, one for starlike functions of order $\alpha$ and one for spirallike functions with parameter $a$. We begin by obtaining a subordination result for each class.

One argument used to obtain the subordination depends on Theorem $A$. We need to know that if $f \in \mathbb{Q}, f(0)=0, f(z) / z \neq 0$, then letting $q(z)=z f^{\prime}(z) / f(z)$ we find that $(d / d z) \log f(z)=[q(z)-1] / z$ and so by integration

$$
f(z)=z \exp \left\{\int_{0}^{z} \frac{q(w)-1}{w} d w\right\} .
$$

Also, the fact that $f(z)=\log (1-z)$ is univalent and convex is used, and this depends on the computation that $\operatorname{Re}\left\{z f^{\prime \prime}(z) / f^{\prime}(z)+1\right\}=\operatorname{Re}\{1 /(1-z)\}>1 / 2>0$, if $|z|<1$.

Lemma 1. If $f \in S t(\alpha)$ then $f(z) / z$ is subordinate to $F(z)=1 /(1-z)^{2-2 a}$ in $\Delta$.

Proof. Suppose that $f \in S_{t}(\alpha)$ so that, according to (11), we may write

$$
\log \frac{f(z)}{z}=\int_{0}^{z} \frac{q(w)-1}{w} d w .
$$

The functions $\{q\}$ consist of those functions subordinate to $q_{0}(z)=a+$ $(1-\alpha)(1+z) /(1-z)$, the function mapping $\Delta$ one-to-one onto the half-plane 
Re $w>$ a so that $q_{0}(0)=1$. The integral in (12) defines a continuous linear operator of order zero on the family $\{q\}$, and so by Robinson's extension of Theorem $A$, we conclude that $\log (f(z) / z)$ is hull subordinate to $G(z)=\int_{0}^{z}\left(q_{0}(w)-1\right) / w d w$. Since $G(z)=-2(1-\alpha) \log (1-z)$ is univalent and convex in $\Delta$ this hull subordination becomes a subordination. This is equivalent to $f(z) / z$ is subordinate to $F(z)$.

Lemma 2. If $f \in S_{p}(\alpha)$ then $f(z) / z$ is subordinate to $F(z)=1 /(1-z)^{\left(1+e^{-2 i a)}\right.}$ in $\Delta$.

Proof. This is similar to the proof of Lemma 1. The functions $\{q\}$ are now subordinate to $q_{0}=e^{-i a}[\cos \alpha(1+z) /(1-z)+i \sin \alpha]$, another one-to-one mapping of $\Delta$ onto a half-plane. We may apply Robinson's result and then drop the hull in the subordination since $\int_{0}^{z}\left(q_{0}(w)-1\right) / w d w=-\left(e^{-2 i a}+1\right) \log (1-z)$ is univalent and convex for $|z|<1$.

Remarks. The result in Lemma 1 was proved by E. Strohhäcker in [16] for $S t$ and for general $a$ by I. S. Jack in [9] using an entirely different method from the one presented above. There is another argument by which Lemmas 1 and 2 can be obtained. The functions in $S_{t}(\alpha)$ are characterized by the formula

$$
f(z)=z \exp \left\{-(2-2 \alpha) \int_{X} \log (1-x z) d \mu(x)\right\}
$$

where $\mu$ is a probability measure on $X=\{x:|x|=1\}$. Lemma 1 is a consequence of formula (13) and the fact that $\int_{X} \log (1-x z) d \mu(x)$ is subordinate to $\log (1-z)$. The last subordination follows from the facts that $\log (1-z)$ is univalent and convex and $\mu$ is a probability measure. In a similar way, Lemma 2 follows from the representation formula

$$
f(z)=z \exp \left\{-\left(1+e^{-2 i a}\right) \int_{X} \log (1-x z) d \mu(x)\right\}
$$

for functions in $S_{p}(\alpha)$.

Lemma 3. Suppose that $g$ is subordinate to the nonconstant function $G$ in $\Delta$ and let $D=G[\{z:|z| \leq r\}](0<r<1)$. If $\left|z_{0}\right| \leq r$ and $g\left(z_{0}\right) \in \partial D$ then $g(z)=$ $G(x z)$ for some complex number $x,|x|=1$.

Proof. Suppose that $w_{0}=g\left(z_{0}\right) \in \partial D,\left|z_{0}\right| \leq r$ and $0<r<1$. To each point $w_{0}$ on $\partial D$ there exists at least one point $z_{1}$ with $\left|z_{1}\right| \leq r$ so that $G\left(z_{1}\right)=w_{0}$. Since $G$ is an open mapping we must have $\left|z_{1}\right|=r$. The subordination implies that $g(z)=G(\phi(z))$ where $\phi \in \mathbb{Q}, \phi(0)=0$ and $|\phi(z)|<1 \quad(|z|<1)[11$, p. 227]. If we set $z_{2}=\phi\left(z_{0}\right)$, then $G\left(z_{2}\right) \in \partial D$. Because of Schwarz's lemma, $\left|z_{2}\right| \leq\left|z_{0}\right|$ $\leq r$ and so by the openness of $G$ we conclude that $\left|z_{2}\right|=r$. Thus, $\left|z_{0}\right|=r$ and 
so $\left|\phi\left(z_{0}\right)\right|=\left|z_{0}\right|$. But the equality $\left|\phi\left(z_{0}\right)\right|=\left|z_{0}\right|$ at any point in $\Delta, z_{0} \neq 0$, implies that $\phi(z)=x z$ and $|x|=1$.

Theorem 3. Suppose that $\Phi$ is a nonconstant entire function and that $0<\left|z_{0}\right|$ $<1$. Then the maximum of either

$$
\operatorname{Re}\left\{\Phi\left[\log \frac{f\left(z_{0}\right)}{z_{0}}\right]\right\} \text { or }\left|\Phi\left[\log \frac{f\left(z_{0}\right)}{z_{0}}\right]\right|
$$

over the class $S_{t}(\alpha)$ is attained only for a function of the form $f(z)=z /(1-x z)^{2-2 a}$, $|x|=1$.

Proof. Each problem has a solution as $S t(\alpha)$ is compact and the functionals are continuous. As pointed out by Golusin in [5], any function which maximizes $\left|\Phi\left[\log \left(f\left(z_{0}\right) / z_{0}\right)\right]\right|$ also maximizes $\operatorname{Re}\left\{e^{i \beta} \Phi\left[\log \left(f\left(z_{0}\right) / z_{0}\right)\right]\right\}$ for a suitable real number $\beta$, and thus we need only consider problems of the first kind.

Suppose that $f \in S_{t}(\alpha)$. Lemma 1 implies that $f(z) / z$ is subordinate to $F(z)=$ $(1-z)^{-(2-2 a)}$, which is the same as $\log [f(z) / z]$ is subordinate to $\log F(z)$. Thus, $g(z)=\Phi[\log (f(z) / z)]$ is subordinate to $G(z)=\Phi[-(2-2 a) \log (1-z)]$. Also, $G$ is nonconstant, since $\Phi$ is nonconstant.

The subordination implies that $g[\{z:|z| \leq r\}] \subset D=G[\{z:|z| \leq r\}]$ (since the associated function $\phi$ satisfies $|\phi(z)| \leq|z|)$ for each $r(0<r<1)$. The function $G(x z),|x| \leq 1$, is subordinate to $G(z)$ and so $\left\{g\left(z_{0}\right): g\right.$ is subordinate to $G$ in $\left.\Delta\right\}$ $=D$ where $r=\left|z_{0}\right|$. This uses the fact that $G(x z)$ is also of the form $f(z) / z$ where $f \in S_{t}(\alpha)$. By considering a support line to the compact set $D$ we conclude that

$$
\max _{f \in S t(\alpha)} \operatorname{Re}\left\{\Phi\left[\log \frac{f\left(z_{0}\right)}{z_{0}}\right]\right\}=\operatorname{Re} w_{1},
$$

where $w_{1} \in \partial D$. Since $G$ is an open mapping there is a number $z_{1}$ so that $\left|z_{1}\right|=r$ and $G\left(z_{1}\right)=w_{1}$. Of all the numbers $w_{1}$ (there are only finitely many) satisfying (15) there is one for which $w_{1}=\Phi\left[\log \left(f\left(z_{0}\right) / z_{0}\right)\right]$ where $f$ is a given solution to the extremal problem. This is the precise situation described by Lemma 3 and so

$$
\Phi[\log (f(z) / z)]=\Phi[-(2-2 \alpha) \log (1-x z)] .
$$

That is, if $f$ is a solution of the extremal problem then (16) holds for some $x$, $|x|=1$.

Since $\Phi$ is nonconstant we may write $\Phi(w)=c_{0}+c_{n} w^{n}+c_{n+1} w^{n+1}+\cdots$ where $c_{n} \neq 0$. If we let $\log (f(z) / z)=a_{1} z+a_{2} z^{2}+\cdots$ and $-(2-2 \alpha) \log (1-x z)=$ $\beta_{1} z+\beta_{2} z^{2}+\cdots$ then (16) implies that $c_{n} \alpha_{1}^{n}=c_{n} \beta_{1}^{n}$. Since $c_{n} \neq 0$ this shows that $\alpha_{1}^{n}=\beta_{1}^{n}$ and so, in particular, $\left|\alpha_{1}\right|=\left|\beta_{1}\right|$. Since $\log (f(z) / z)$ is subordinate 
to $-(2-2 \alpha) \log (1-x z)$ the equality $\left|\alpha_{1}\right|=\left|\beta_{1}\right|$ is only possible (note that $\left.\beta_{1}=2-2 \alpha \neq 0\right)$ if $\log (f(z) / z)=-(2-2 \alpha) \log (1-x y z)$ for some $y,|y|=1$. Therefore, we conclude that $f(z)=\cdot z /(1-u z)^{2-2 a},|u|=1$, if $f$ is a solution to the extremal problem.

Theorem 4. Suppose that $\Phi$ is a nonconstant entire function and that $0<$ $\left|z_{0}\right|<1$. Then the maximum of either

$$
\operatorname{Re}\left\{\Phi\left[\log \frac{f\left(z_{0}\right)}{z_{0}}\right]\right\} \text { or }\left|\Phi\left[\log \frac{f\left(z_{0}\right)}{z_{0}}\right]\right|
$$

over the class $S_{p}(\alpha)$ is attained only for a function of the form $f(z)=$ $z /(1-x z)^{1+e^{-2 i a}},|x|=1$.

Proof. The proof of this theorem is the same as that given for Theorem 3, except we appeal to Lemma 2 instead of Lemma 1.

Remarks. 1. Theorems 3 and 4 may be equivalently expressed in the form of maximizing $\operatorname{Re}\left\{\Phi\left[f\left(z_{0}\right) / z_{0}\right]\right\}$. This follows since the two sets of functions $\{\Phi[\log (f(z) / z)]\}$ and $\{\Phi[f(z) / z]\}$ with varying entire functions $\Phi$ are the same, because $f(z) / z \neq 0$ in $\Delta$.

2. Undoubtedly there are a variety of families of analytic functions for which results like Theorems 3 and 4 hold. For example, this is the case for $k$-fold symmetric functions in $S_{t}(\alpha)$ or in $S_{p}(\alpha)$. The hypotheses of $S_{t}(\alpha)$ and $S_{p}(\alpha)$ could be combined to obtain such assertions for the normalized functions which satisfy $\operatorname{Re}\left\{e^{i a} z f^{\prime}(z) / f(z)\right\}>\beta$.

A result which is equivalent to Theorem 3 may be stated for the class $K(\alpha)$. If we set

$$
F(z)=\left\{\begin{array}{l}
\frac{1}{1-2 a}\left[\frac{1}{(1-z)^{1-2 a}}-1\right], \text { if } a \neq 1 / 2, \\
-\log (1-z), \text { if } \alpha=1 / 2,
\end{array}\right.
$$

then the maximum of $\operatorname{Re}\left\{\Phi\left[\log f^{\prime}\left(z_{0}\right)\right]\right\}$ over $K(\alpha)$ occurs only for the functions $x^{-1} F(x z),|x|=1$. This simply uses the fact that $f \in K(\alpha)$ if and only if $z f^{\prime}(z) \epsilon$ $S_{t}(\alpha)$.

The maximum of $\operatorname{Re}\left\{\Phi\left[\log \left(f\left(z_{0}\right) / z_{0}\right)\right]\right\}$ over $K(\alpha)$ is also attained only by the functions $x^{-1} F(x z)$, if $a \geq 1 / 2$. This can be proved by our method, since $f(z) / z$ is subordinate to $F(z) / z$ for each $f$ in $K(\alpha)$ when $\alpha \geq 1 / 2$, as shown in [3]. This subordination is likely to hold for all $a$, and, if so, it would provide the above result on extremal problems over $K(\alpha)$ for each $\alpha$. 
3. There are, of course, relationships between the two parts of this paper. For example, if each function $g$ in a family $\mathcal{G}$ is hull subordinate to $g_{0}$, then $g_{0}$

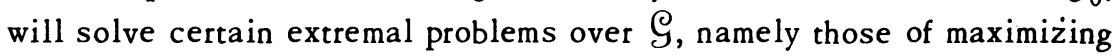
$\max |z|=r \operatorname{Re} e^{i \theta} g(z)$. Given the hypotheses of Theorem 1, this asserts that

$$
\max _{f \in \mathfrak{Y}} \max _{|z|=r} \operatorname{Re} e^{i \theta} \mathcal{Q}[f(z)]=\max _{|z|=r} \operatorname{Re} e^{i \theta \varrho}\left[f_{0}(z)\right] .
$$

More generally, in order to maximize $\max |z|={ }_{r} \operatorname{Re} e^{i \theta \varrho}[f(z)]$ over $\mathcal{F}$ it suffices to find the maximum over $\left(\mathcal{E} \mathcal{F} \mathcal{F}\right.$. If we seek to maximize $\operatorname{Re} e^{i \theta \mathscr{L}}\left[f\left(z_{0}\right)\right]$ over $\mathcal{F}$ where $z_{0}$ is fixed $\left(\left|z_{0}\right|<1\right)$ and $\mathcal{L}$ is a continuous linear operator, this can be resolved by the more general result: if $J$ is a continuous, linear functional on $\mathfrak{A}$

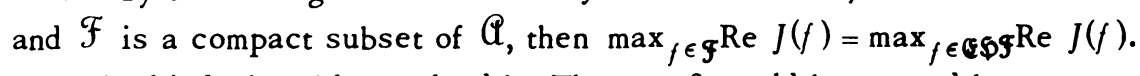

The kind of problem solved by Theorem 3 could be treated by extreme-point theory if the extreme points of the family $\{\Phi[f(z) / z]\}$ could be determined. Except in the case that $\Phi$ is a linear function, generally we cannot expect to determine this set of extreme points.

However, the extreme points of this family can be found when $f$ varies in $S_{t}(\alpha)$ (and $S_{p}(\alpha)$ ) with the specific functions $\Phi(w)=A+B w^{p}$, where $p>0$. To show this, we note that formula (13) implies that each function in $S_{t}(\alpha)$ can be uniformly approximated on $|z| \leq r(0<r<1)$ by functions of the form

$$
g(z)=z \prod_{k=1}^{n} \frac{1}{\left(1-x_{k} z\right)^{\lambda_{k}(2-2 a)}},
$$

where $\left|x_{k}\right|=1, \lambda_{k} \geq 0$ and $\sum_{k=1}^{n} \lambda_{k}=1$. Because of the result [3, Theorem 1], we may write

$$
\left[\frac{g(z)}{z}\right]^{p}=\prod_{k=1}^{n} \frac{1}{\left(1-x_{k} z\right)^{p \lambda_{k}(2-2 \alpha)}}=\int_{X} \frac{1}{(1-x z)^{q}} d \mu(x),
$$

where $q=\sum_{k=1}^{n} p \lambda_{k}(2-2 \alpha)=p(2-2 \alpha)$ and $\mu$ is a probability measure on $X=$ $\{x:|x|=1\}$. This implies that the set functions $G$ representable by $G(z)=$ $\int_{X} 1 /(1-x z)^{q} d \mu(x), q=p(2-2 \alpha)$, with varying $\mu$ is the closed convex hull of the family $\left\{[f(z) / z]^{p}\right\}, f \in S_{t}(\alpha)$. The mapping $\mu \rightarrow G$ is readily shown to be one-to-one and thus the extreme points of this hull are precisely the functions $g(z)=1 /(1-x z)^{q},|x|=1$ (see $[2$, Theorem 1]). This obtains the extreme points for the hull of the family $\left\{A+B[f(z) / z]^{p}\right\}, f \in S_{t}(\alpha)$. In particular, the result of Theorem 3 can also be proved by extreme-point theory if $\Phi(w)=A+B w^{p}$.

This last assertion is, of course, less general than Theorem 3, and is not as "elementary" as our proof of that theorem. Although we have proved Theorem 4 in this same way we cannot assert that Theorem 1 is applicable to the class $S_{p}(\alpha)$. 
An open problem is the determination of $S_{2} S_{p}(\alpha)$ and $\mathbb{E} S_{2} S_{p}(\alpha)$, although Theorem 4 and other results about $S_{p}(\alpha)$ suggest an obvious conjecture.

\section{REFERENCES}

1. D. A. Brannan, J. G. Clunie and W. E. Kirwan, On the coefficient problem for functions of bounded boundary rotation, Ann. Acad. Sci. Fenn. Ser. AI (to appear).

2. L. Brickman, T. H. MacGregor and D. R. Wilken, Convex hulls of some classical families of univalent functions, Trans. Amer. Math. Soc. 156 (1971), 91-107. MR 43 \#494.

3. L. Brickman, D. J. Hallenbeck, T. H. MacGregor and D. R. Wilken, Convex hulls and extreme points of families of starlike and convex mappings, Trans. Amer. Math. Soc. (to appear).

4. N. Dunford and J. T. Schwartz, Linear operators. I: General theory, Pure and Appl. Math., vol. 7, Interscience, New York, 1958. MR 22 \#8302.

5. G. M. Goluzin, On a variational method in the the ory of analytic functions, Leningrad. Gos. Univ. Učen. Zap. 144, Ser. Mat. Nauk 23 (1952), 85-101; English transl., Amer. Math. Soc. Transl. (2) 18 (1961), 1-14. MR 17, 1070; MR 23 \#A1803.

6. D. J. Hallenbeck, Convex hulls and extreme points of some families of univalent functions, Ph.D. Dissertation, State University of New York, Albany, N. Y., 1972.

7. D. J. Hallenbeck and T. H MacGregor, Subordination and extreme-point theory, Pacific J. Math. (to appear).

8. D. J. Hallenbeck, Convex hulls and extreme points of some families of univalent functions, Trans. Amer. Math. Soc. (to appear).

9. I. S. Jack, Functions starlike and convex of order a, J. London Math. Soc. (2) 3 (1971), 469-474. MR 43 \#7611.

10. W. E. Kirwan, A note on extremal problems for certain classes of analytic functions, Proc. Amer. Math. Soc. 17 (1966), 1028-1030. MR 34 \# 2854.

11. Z. Nehari, Conformal mapping, McGraw-Hill, New York, 1952. MR 13, 640.

12. M. S. Robertson, On the the ory of univalent functions, Ann. of Math. (2) 37 (1936), 374-408.

13. - An extremal problem for functions with positive real part, Michigan Math. J. 11 (1964), 327-335. MR 30 \#243.

14. R. M. Robinson, Univalent majorants, Trans. Amer. Math. Soc. 61 (1947), 1-35. MR 8, 370.

15. L. Špaček, Contribution à la théorie des fonctions univalentes, Časopis Pěst. Mat. 62 (1932), 12-19.

16. E. Strohhäcker, Beiträge zur Theorie der schlichten Funktionen, Math. Z. 37 (1933), 356-380.

DEPARTMENT OF MATHEMATICS, STATE UNIVERSITY OF NEW YORK AT ALBANY, ALBANY, NEW YORK 12222 